\title{
Fibrous dysplasia of Sphenoid bone
}

\author{
Harjitpal Singh $\cdot$ C. Mohan
}

\begin{abstract}
Fibrous dysplasia represents a disturbance of normal bone development specifically a defect in osteoblastic differentiation and maturation that originates in the mesenchymal precursor of the bone. In fibrous dysplasia, the lesion expands, which leads to a distortion and weakening of bone. Although the lesion is not encapsulated, it tends to remain enclosed within a shell of cortical bone. This shell can be thinned as a result of the pressure exerted on it. As it slowly progresses, fibrous dysplasia can cause skeletal destruction and deformity.
\end{abstract}

Keywords Fibrous dysplasia

\section{Introduction}

Fibrous dysplasia is an uncommon benign disorder of unknown etiology. It is a non-neoplastic, primary disorder of bone. The disease process replaces normal medullary bone with a variable amount of abnormal and structurally weak fibrous and osseous tissue Fibrous dysplasia is associated with a defect in osteoblastic differentiation and maturation that originates in the mesenchymal precursor of the bone. Fibrous dysplasia is of particular interest to the otolaryngologist because of its predilection for the facial and

\author{
Harjitpal Singh $(\square)$ \\ Junior resident, \\ C. Mohan \\ Prof \& Head ENT Dept.,
}

IGMC Shimla - 171001

Himachal Pradesh cranial bones, where it causes deformity and dysfunction. In this paper, we report the case of a patient with fibrous dysplasia of the sphenoid bone with proptosis and causing slight midline shift of the brain parenchyma.

\section{Gase Report}

A 34-year-old male attended ENT OPD with complaints of protrusion of left eye for 9 years (Fig. 1). His record showed that he was diagnosed as a case of fibrous dysplasia of sphenoid at that time and as he didn't have any other symptom associated with, he was put under observation. Now he complained that eye protrusion had increased, but still there was no other complaint. His vision was fully normal. There was no sign or symptom of increased intracranial tension. His nasal examination was within normal limits.

CT Scan of orbit and brain showed that there was a lytic expansile lesion involving left sphenoid bone, extending into left orbit. It was pushing left eye ball and posterior wall of left maxilla anteriorly. The growth showed irregular sclerosis and its inner contents were cystic. There was no enhancement on IV contrast (Fig. 2). Provisional diagnosis of fibrous dysplasia was made on CT finding. Because of position of the lesion and its slow growth without causing much symptoms, it was decided to keep patient under observation with consultation with neurosurgeon.

\section{Discussion}

In 1937, McCune and Bruch first suggested that of all the abnormalities of bone formation, this disorder should have its own place as a distinct clinical entity ${ }^{1}$. The following year, Lichtenstein introduced the term fibrous dysplasia ${ }^{1}$. There are three categories of the disease:

1. Monostotic fibrous dysplasia involves a single bone and represents $70 \%$ of all cases. Commonly involves femur, tibia, or facial bones. It generally becomes arrested by puberty. There is an equal male to female ratio 
Fig. 1 Patient with (L) proptosis

Fig. 2 Contrast CT (L) orbit showing no enhancement.
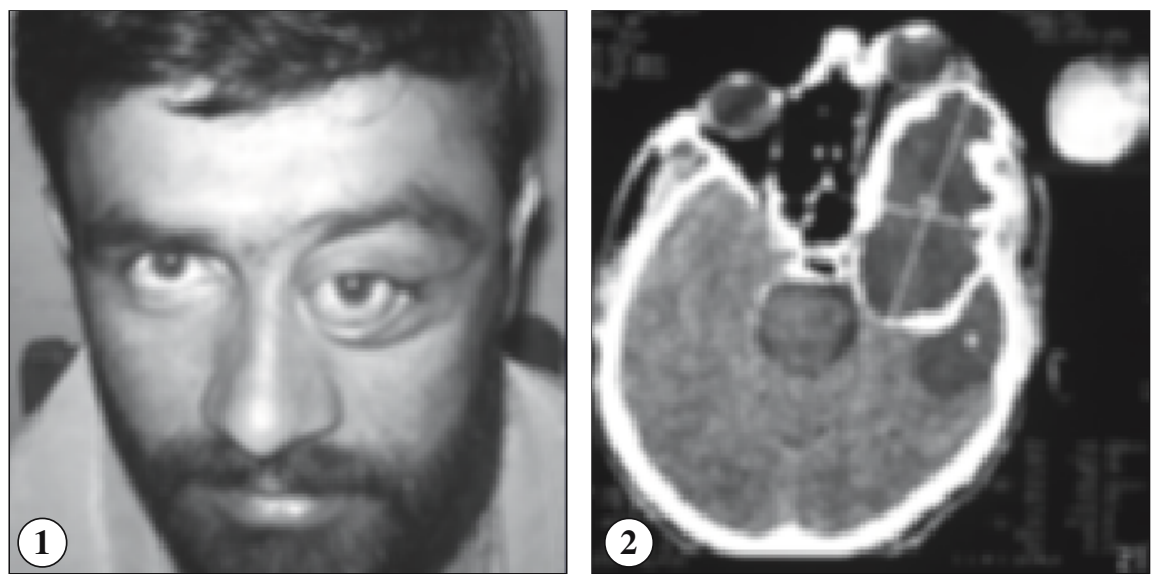

2. Polyostotic fibrous dysplasia involves multiple bones most frequently of the lower limbs. In the skull sphenoid and frontal bones are affected. These lesions may be localized to one region of the body or they may be disseminated, involving virtually every bone. There is a female predilection in polyostotic fibrous dysplasia, and up to 50\% may involve bones in the head and neck. These lesions are more likely to continue to progress even after puberty, beyond the third or fourth decades.

3. McCune-Albright syndrome is seen in approximately $3-5 \%$ of patients with fibrous dysplasia. In this the bony involvement is associated with sexual precocity, hyperthyroidism and café-au-lait macular spots. It primarily affects females. A possible relationship between fibrous dysplasia and primary hyperparathyroidism has been suggested ${ }^{[2]}$.

Skull involvement occurs in $27 \%$ of monostotic and up to $50 \%$ of polyostotic patients. Fibrous dysplasia involving the face and skull is called "Leontiasis ossea." Without treatment, one or more bones progressively increase in size, and move into the cavities of the eye, mouth, and/or the nose and its sinuses. Also, abnormal protrusion of the eyeball (exophthalmos) may develop and eventually cause complete loss of sight because it presses on the optic nerve.

The mode of transmission has not yet been established. If it is genetic, it is unclear whether fibrous dysplasia has an autosomal-dominant or an autosomal-recessive character. It has been suggested that monostotic fibrous dysplasia occurs secondarily to an arrest of bone maturation ${ }^{3}$. It has also been proposed that fibrous dysplasia might be associated with increased levels of steroid hormone receptors (receptors of estrogens or progesterone).

Fibrous Dysplasia of nasal cavity and paranasal sinuses presents with facial asymmetry along with painless swelling in cheek or orbital regions or frontal headache ${ }^{4}$. The enlargement of facial bones is painless, usually noticed in adolescence and may continue after somatic growth has ceased. Initial symptoms usually appear during childhood or early adolescent- a period of active skeletal growth.

When fibrous dysplasia of the frontal (forehead bone) and/ or sphenoid (bone at the base of the skull) bones progresses, these bones become thick and dense. This increase in size eventually causes the facial features and skull to become misshapen. In these cases more than one bone is usually involved. It can also result in cranial nerve problems.

Isolated lesion of sphenoid sinus is a rare entity ${ }^{5}$, as polyostotic lesions involve multiple bones. In this case also there is isolated involvement of left sphenoid bone which was extending into left orbit, rest of facial bones or sinuses were normal. Lawrence R Lustig et al in their study of 21 cases of fibrous dysplasia of facial bones had found involvement of sphenoid in $43 \%$ patients $^{6}$. But in all these cases there was multiple bone involvement, not even a single case of isolated lesion of sphenoid sinus was found. They performed orbital decompression in 5 cases who presented with proptosis and diplopia. In rest of cases nothing particular was done for sphenoid involvement.

Faraci RP \& Ketcham AS reported a case of isolated sphenoid lesion in 13-year-old black male ${ }^{7}$. They reported that one year following admission, the patient's proptosis disappeared and his symptoms were markedly improved.

\section{Radiographic features.}

The radiologic features reflect the morphology of the disease and vary with the amount of fibrosis and calcification. Three patterns of fibrous dysplasia of the skull and facial bones have been described ${ }^{8}$.

1 The pagetoid, or "ground-glass," pattern is the most common ( $56 \%$ of all cases); it appears as a mixture of dense and radiolucent areas of fibrosis.

2 The sclerotic pattern (23\% of cases) is uniformly dense.

3 The cystic pattern (21\%) is characterized by a spherical or ovoid lucidity surrounded by a dense bony shell. 
But these classic radiographic appearances are based on the plain films which have little relevance these days, in era of CT \& MRI imaging. Computed tomography is the investigation of choice for diagnosis and follow-up because of its superior bony details and accurate assessment of the extent of the lesion ${ }^{6}$. Furthermore, CT can often assist in differentiating fibrous dysplasia from other osteodystrophies of the skull base, including otosclerosis. osteogenesis imperfecta, Paget' disease and osteopetrosis. Distinguishing features of fibrous dysplasia on CT includes "ground-glass" appearance, symmetry, thickness of cranial cortices, and presence of cystlike changes. There is often clear margin between affected and unaffected bone.

MRI is an additional useful modality that can help distinguish fibrous dysplasia from meningioma, osteoma, or mucocele and define the extent of soft tissue involvement, particularly if central nervous system structures are impinged on.

Ming-Tsung Chen et al studied role of bone scintigraphy in evaluating the extent of the disease in fibrous dysplasia cases and found it to be a single and cheap examination comparing with other imaging methods ${ }^{9}$.

Operative procedures should focus on three directives: the restoration of function, the prevention of complications, and the restitution of cosmesis.

Spontaneous transformation to malignancy in fibrous dysplasia has been reported in $0.5 \%$ of patients ${ }^{1}$. Osteosarcoma is the most common malignancy, followed by chondrosarcoma, fibrosarcoma, and giant cell sarcoma ${ }^{1}$. The average length of time between the diagnosis of fibrous dysplasia and a malignant transformation is 13.5 years. Radiographic features suggestive of malignant degeneration include a rapid increase in the size of the lesion and a change from a previously mineralized bony lesion to a lytic lesion. Clinical findings of increasing pain and an enlarging soft tissue mass suggest malignant change.

\section{References}

1. Yagoda MR, Selesnick SH. Temporal bone fibrous dysplasia and cholesteatoma leading to the development of a parapharyngeal abscess. J Laryngol Otol 1994; 108:51-53.

2. Caudill R, Saltzman D, Gaum S, Granite E. Possible relationship of primary hyperparathyroidism and fibrous dysplasia: Report of a case. J Oral Surg 1977; 35:483-490.

3. El Deeb M, Waite DE, Gorlin RJ. Congenital monostotic fibrous dysplasia-a new possibly autosomal recessive disorder. J Oral Surg 1979;37:520-525.

4. FuYao-Shi, Perzin Karl H. Non-epithelial tumors of the Nasal cavity, Paranasal sinuses and Nasopharynx: A ClinicoPathologic study. II. Osseous and Fiobrosseous Lesions including Osteoma, Fibrous Dysplasia, Ossifying Fibroma, Osteoblastoma, Giant Cell Tumour and Osteosarcoma. Cancer 1974; 33: 1289-1305.

5. Dharambir S Sethi. Isolated sphenoid lesions: Diagnosis and management. Otolaryngol Head Neck Surg 1999; 120:730-736.

6. Lawrence R. Lustig, Michael J Holliday, Edward F. McCarthy, George T Nager. Fibrous dysplasia involving the skull base and temporal bone. Arch Otolaryngol Head Neck Surgery 2001; 127: $1239-1247$.

7. Faraci RP \& Ketcham AS. Fibrous dysplasia of the sphenoid sinus in an adolescent male. J Surg Oncol. 1975;7(6):461-465.

8. Brown EW, Megerian CA, McKenna MJ, Weber A. Fibrous dysplasia of the temporal bone: Imaging findings. MR Am J Roentgenol 1995; 164:679-682.

9. Ming-Tsung Chen, Yen-Kung Chen, Chi-Tai Ku, Su-Cheng Wang, Yeh-You Shen. 18F-FDG PET and 99mTc-MDP Findings in McCune-Albright Syndrome.Ann Nucl Med Sci. 2003;16:143-146 